\title{
Functional outcome in conservatively treated non-displaced scaphoid fractures ${ }^{*}$
}

\author{
Tessa Drijkoningen $^{1 \# \dagger}$, Frank J. P. Beeres ${ }^{1 \#}$, Roderick H. van Leerdam ${ }^{1 \#}$, Daan Ootes ${ }^{1}$, \\ Diana C. Grootendorst ${ }^{2}$, Marleen Otoide-Vree ${ }^{2 \#}$, Steven J. Rhemrev ${ }^{1 \#}$ \\ ${ }^{1}$ Department of Trauma Surgery, Medisch Centrum Haaglanden, Hague, The Netherlands \\ ${ }^{2}$ Landsteiner Instituut, Medisch Centrum Haaglanden, Hague, The Netherlands \\ Email: ${ }^{\dagger}$ tessa.drijkoningen@gmail.com
}

Received 21 September 2012; revised 23 October 2012; accepted 10 November 2012

\begin{abstract}
Purpose: This study evaluated the functional outcome after conservative treatment of non-displaced scaphoid fractures using an international validated outcome scale (DASH). Methods and materials: Between 2005 and 2010, 60 patients with a non-displaced scaphoid fracture were included. When a patient visited the emergency department and was clinically suspected of a scaphoid fracture radiography was performed. If no fracture was diagnosed with this modality a CT or MRI-scan both in combination with bonescintigraphy was performed. Patients with scaphoid fractures diagnosed with CT/MRI and bonescintigraphy were treated with a six-week scaphoid forearm cast. Within 1 year after cast removal patients filled in the DASH questionnaire. Results: Sixty (80\%) patients returned the DASH questionnaire at 12 months after treatment. Thirty-eight $(63.3 \%)$ were male and the mean age was 35 (range 11 - 83). Forty-four (73.3\%) patients had a mid-waist fracture of the scaphoid, $13(21.7 \%)$ had a fracture of the distal pole and three $(0.05 \%)$ had a proximal fracture. Median DASH score at one year after the trauma was 6 (range 3 - 15) for patients with a distal pole fracture and 5 (range 0 - 21.5) for mid-waist fractures $(p=0.7$, Table 2). For the three patients with a proximal scaphoid fracture the DASH scores appeared higher and were 83, 82 and 30 respectively. Conclusion: Conservative treatment for six weeks with a below the elbow cast is sufficient for the majority of patients with an occult distal or mid-waist scaphoid fracture and re-
\end{abstract}

\footnotetext{
*Competing interests: the authors declare that they have no competing interests.

\#Author's contributions: SR, RHVL and TD carried out the study and drafted the manuscript. DO assessed all forms, collected the data and helped draft the manuscript. SAGM participated in the design of the study and MOV performed the statistical analysis. FJPB participated in the design and coordination and helped to draft the manuscript. All authors read and approved the final manuscript.

${ }^{\dagger}$ Corresponding author.
}

sults in a good functional outcome according to the DASH questionnaire.

Keywords: DASH; Scaphoid Fracture; Os Scaphoideum; Hand Trauma; Conservative Treatment

\section{INTRODUCTION}

The scaphoid bone is the most common broken carpal bone. Scaphoid fractures are reported to represent $2 \%$ $7 \%$ of all fractures and over $70 \%$ of all hand fractures presenting to accident and emergency departments [1]. Seventy percent of all scaphoid fractures are through the waist of the bone. In general, the prognosis of distal fractures of the scaphoid is often better than that for proximal fractures because of the retrograde blood supply [2]. In all scaphoid fractures early diagnosis is imperative owing to potential complications following the fracture, including non-union, avascular necrosis, carpal instability and osteoatrosis.

Despite the common occurrence of scaphoid fractures, the treatment remains an on-going debate [3]. Centres of expertise achieve excellent results with surgical treatment, but conservative treatment is also proven effective with consolidation rates exceeding 85\% [4,5]. Vinnars et al. [6] described no differences in outcome scores between operative treatment and non-operative treatment. Success of any treatment can be measured using biological outcome but also using functional outcome. For patients, functional outcome in daily life is more important. A joint initiative of the American Academy of Orthopaedic Surgeons (AAOS), the Council of Musculoskeletal Speciality Societies (COMSS) and the institute for Work and Health (Toronto, Ontario) designed the Disabilities of Arm Shoulder and Hand (DASH) Outcome Measurequestionnaire. This questionnaire measures functional outcome and provides a validated and subjective "general functional outcome assessment" [6]. The DASH is often used for the wrist, but it was especially designed to 
assess the function of the whole upper limb.

The aim of this study is to evaluate functional outcome after conservative treatment of non-displaced scaphoid fractures using an international validated outcome scale (DASH). In addition, we studied whether fracture location is associated with functional outcome.

\section{MATERIALS AND METHODS}

\subsection{Patients}

All patients with acute non-displaced scaphoid fractures diagnosed by radiographs, Magnetic Resonance (MR) or Computed Tomography (CT) or bonescintigraphy were included after written informed consent. Exclusion criteria were displaced scaphoid fractures, ipsilateral fractures, secondary operated proximal scaphoid fractures and fractures of the scaphoid in the medical history. The Medical Ethics Committee gave medical and ethical clearance for this prospective cohort study.

\subsection{Study Design}

Patients attending our institution for the period between 2005 and 2010 were included in this prospective followup study. They were asked to fill in the DASH questionnaire and return it by mail 1 year after cast removal. Fractures were classified according to the Mayo and Herbert classification systems [7]. The Mayo classification described fractures based on location and distinguishes between proximal, waist and distal fractures. The Herbert Classification relates to radiologic appearance of fracture instability.

\subsection{DASH}

Functional outcome after scaphoid fracture was assessed by the DASH questionnaire assessing upper limb function. The DASH questionnaire consists of a 30-question module on functional disabilities and two 4-question optional modules on sports and work respectively. Each question on disability is scored on a scale from 1 to 5 , where 1 represents no difficulties and/or no residual symptoms and 5 represents functional inability or extreme symptoms. The DASH score was calculated by the following formula: ((sum of $n$ responses) -1$) \times 25) / n$. A low DASH score indicates fewer functional disabilities and a high DASH score indicates functional disability or persistent symptoms. Hence, no disability equals a DASH outcome of zero and total disability equals to a DASH outcome of 100 .

\subsection{Diagnosis and Treatment}

For the definite diagnosis and treatment the following flow chart was used: when a patient visited the emergency department and was clinically suspected of a scaphoid fracture radiography was performed. If no fracture was diagnosed with this modality a CT or MRI-scan both in combination with bone scintigraphy was performed. Patients with scaphoid fractures diagnosed with either imaging method were treated with a six-week scaphoid forearm cast below the elbow incorporating the thumb as far as its interphalangeal joint. If no fracture was diagnosed patients visited the physician two weeks after first presentation for clinical examination and radiography. When a scaphoid fracture was diagnosed on radiography at this point a cast was given for 4 weeks. For all patients was that if they had persisting clinical signs 6 weeks after the trauma which includes pain in the anatomic snuffbox and pain upon axial compression of the first and/or second digit of a scaphoid fracture or no signs of radiologycal consolidation, a new cast was applied for an additional two weeks. This procedure was repeated every two weeks for a maximum of 12 weeks until radiological evidence of consolidation and no clinical signs of fracture were left.

\subsection{Statistics}

Data entry and analysis were performed with Statistical Packages for Social Sciences (SPSS) version 17.0 for Windows. As data were not normally distributed, median with their 25 - 75 percentiles were given and differences were tested using the Wilcoxon rank sum test at the $5 \%$ significance level.

\section{RESULTS}

Seventy-five patients with an occult scaphoid fracture were included between 2005 and 2010. Sixty (80\%) patients returned the DASH questionnaire at 12 months after cast removal. Ten patients were untraceable, one died and four partially completed follow-up or denied further participation. Of the 60 patients with complete data 38 (63.3\%) were male and the mean age was 35 years (range $11-83$ ).

Forty-four (73.3\%) patients had a mid-waist fracture of the scaphoid, $13(21.7 \%)$ had a fracture of the distal pole and three $(0.05 \%)$ had a proximal fracture (Table 1). The median cast duration in patients with a distal pole and a mid-waist scaphoid fracture was 6 weeks (Table 2).

Median DASH score at one year after the trauma was 6 (range 3 - 15) for patients with a distal pole fracture and 5 (range $0-21.5)$ for mid-waist fractures $(\mathrm{p}=0.7$, Table 2). For the three patients with a proximal scaphoid fracture the DASH scores appeared higher and had individual scores of 83, 82 and 30.

\section{DISCUSSION}

The principal findings of this study show that the major- 
ity of patient with a scaphoid fracture have the fracture located in the mid-waist of the scaphoid. Following conservative treatment with a cast for six weeks, all patients with either mid-waist or distal pole fractures in the scaphoid have a good functional outcome, as measured by the DASH questionnaire. Functional outcome was impaired in patients with a proximal pole scaphoid fracture, although this group consisted of only 3 patients.

To date, our study is the only and largest study describing functional outcome of conservatively treated scaphoid fractures. In addition we evaluated the outcome according to the Mayo and Herbert classification and using the DASH-questionnaire. Functional outcome at one year after trauma was comparable between patients with either mid-waist or distal pole scaphoid fracture.

Our data are in line with Forward et al. [8] who studied the clinical outcome using DASH after one year in 42 patients with a scaphoid fracture receiving non-operative treatment. They showed no difference in DASH score between patients with mal-union and no mal-union. They found a median DASH-score of 5 in both groups, comparable with our results. This shows that functional outcome is the same with conservative treatment (DASH) even if patients have a mal-union.

Table 1. Patient characteristics of 60 conservatively treated scaphoid fractures.

\begin{tabular}{lc}
\hline & $\begin{array}{c}\text { Number (\%) } \\
\text { N = 60 }\end{array}$ \\
\hline Meanage & 35 \\
Sex & \\
Male & $38(63 \%)$ \\
Female & $22(37 \%)$ \\
Affected hand & \\
Left & $32(53 \%)$ \\
Right & $28(47 \%)$ \\
& \\
Type of injury & \\
Low energy & $58(97 \%)$ \\
High energy & $2(3 \%)$ \\
Place of fracture & \\
Proximal & $3(5 \%)$ \\
Mid-waist & $44(73 \%)$ \\
Distal & $13(22 \%)$ \\
\hline
\end{tabular}

Table 2. Functional outcome after conservative treatment for distal pole and mid waist scaphoid fractures.

\begin{tabular}{lccc}
\hline & $\begin{array}{c}\text { Distal pole } \\
\mathrm{N}=13\end{array}$ & $\begin{array}{c}\text { Mid-waist } \\
\mathrm{N}=44\end{array}$ & \\
\cline { 2 - 3 } & Median $(25-75$ percentile $)$ & \\
\hline Age (years) & $22(19.5-31)$ & $31(21-54.5)$ & 0.16 \\
Cast duration (weeks) & $6(5.5-8)$ & $6(6-8)$ & 0.71 \\
DASH (points) & $6(3-15)$ & $5(0-21.5)$ & 0.70 \\
\hline
\end{tabular}

A systematic review by Buijze et al. [9] showed that surgical treatment of non- and minimally displaced scaphoid fractures, resulted in significantly better patient reports of functional outcome, satisfaction, grip strength and shorter time of work. Arguments for surgery are the intra-operative anatomical reduction and the drawbacks of a prolonged cast immobilisation. However the benefits of surgical treatment of scaphoid fractures were shown to be short term without long term risk of complications. Disadvantages of long immobilisation period, like with plaster cast are not only related to the socio-economic consequences but also to the atrophy of the forearm and arthrosis of the wrist. In addition, pain, range of motion, non-union and mal-union rates, complications and treatment costs seemed not different between conservative and surgical treatment. This study shows that with conservative treatment of 6 weeks patients with mid-waist and distal pole fractures have a good functional outcome, suggesting that in patients with these type of fractures surgery does not seem to be necessary.

At present, common practice is to treat scaphoid fractures with a plaster cast for more than 11 weeks, although optimal treatment of non-displaced scaphoid fractures is still under discussion. The results of treatment of nondisplaced scaphoid fractures are often determined using the non-union rate. Early studies showed non-union rates between $5 \%-18 \%$ for conservatively treated scaphoid fractures and between 0 - 5\% for surgically treated scaphoid fractures [5,10-13].

This study has potential limitations. First, the study size is relatively small, not allowing multivariate analyses. Age and cast duration were not different between patients with a mid-waist and distal pole fracture of the scaphoid. Moreover, it is unlikely that age and cast duration influenced the DASH score as independent predicttors. This study included only three patients with proximal pole fractures, which is too small to study this group of patients separately. Secondly, follow-up was not complete in $20 \%$ of the study population. The majority of this group consisted of young men failing to attend follow-up. However, it is likely that patients without complaints were less inclined to fill out questionnaires. With this in mind the study would rather have overestimated the DASH score. Finally, the DASH questionnaire is one of the most used worldwide to assess functional outcome after bone fractures. However, the applicability of the questionnaire in patients with scaphoid fractures can be questioned. Rather than being injury specific, DASH is a general assessment tool which does not take into account specific location of the fracture such as dominant or nondominant hand. Specific outcome measurements for injuries of the scaphoid have yet to be designed, hence a general outcome measure was used.

How can the present results be explained? An important 
factor for predicting the functional outcome of a scaphoid fracture is vitality and vascularity of the scaphoid bone. Shown in this study is that patients with mid-waist and distal pole fractures, treated with plaster cast for a median period of 6 weeks, have a good functional outcome measured with the DASH. The 3 patients with a proximal scaphoid fractures had a slow healing process and more functional problems, as is suggested by the high DASH scores and non-optimal functional outcome in our small group of patients with proximal fracture of the scaphoid. This may be because blood supply of this fragment is impaired so union is slow or absent. Therefore, the location of a fracture can strongly influence the process of fracture healing suggesting the proximal pole fractures need a different kind of treatment. In these fractures non-union rates are higher because fracture ends are joined by dense scar tissue and in some cases even a false joint may develop. We presume that midwaist and distal pole fractures need the same treatment whereas proximal pole fractures may need different treatment. Indeed Geissler et al. [14] have shown there is a consensus in literature, in non-union of proximal pole fractures, that surgical treatment is needed.

In conclusion conservative treatment for six weeks with a below the elbow cast is sufficient for the majority of patients with an occult distal or mid-waist scaphoid fracture and results in a good functional outcome according to the DASH questionnaire. Further studies of adequate sample size are needed on functional outcome of the rare proximal pole fracture in the scaphoid and to measure the functional long term outcome.

\section{REFERENCES}

[1] Rhemrev, S.J., de Zwart, A.D., et al. (2010) Early computed tomography compared with bone scintigraphy in suspected scaphoid fractures. Clinical Nuclear Medicine, 35, 931-934. doi:10.1097/RLU.0b013e3181f9de26

[2] Buijze, G.A., Mallee, W.H., et al. (2011) Diagnostic performance tests of suspected scaphoid fractures differ with conventional and latent class analysis. Clinical Orthopedics and Related Research, 469, 3400-3407. doi:10.1007/s11999-011-2074-9
[3] Beeres, F.J., Hogervorst, M., et al. (2005) Outcome of routine bone scintigraphy in suspected scaphoid fractures. Injury, 36, 1233-1236. doi:10.1016/j.injury.2005.02.009

[4] Clay, N.R., Dias, J.J., et al. (1991) Need the thumb be immobilised in scaphoid fractures? A randomised prospective trial. The Journal of Bone \& Joint Surgery, 73, 828-832.

[5] Lindstrom, G. and Nystrom, A. (1990) Incidence of posttraumatic arthrosis after primary healing of scaphoid fractures: A clinical and radiological study. Journal of Hand Surgery, 15, 11-13.

[6] Vinnars, B., Pietreanu, M., et al. (2008) Nonoperative compared with operative treatment of acute scaphoid fractures. A randomized clinical trial. The Journal of Bone \& Joint Surgery, 90, 1176-1185. doi:10.2106/JBJS.G.00673

[7] Green, D.P. (1999) Operative Hand Surgery, 4th Edition, Churchill Livingstone, New York.

[8] Vinnars, D.P., Singh, H.P., et al. (2009) The clinical outcome of scaphoid fracture malunion at 1 year. Journal of Hand Surgery, 34E, 40-46.

[9] Buijze, G.A., Doornberg, J.N., et al. (2010) Surgical compared with conservative treatment for acute nondisplaced or minimally displaced scaphoid fractures: A systematic review and meta-analysis of randomized controlled trials. The Journal of Bone \& Joint Surgery, 92, 1534-1544. doi:10.2106/JBJS.I.01214

[10] Haddad, F.S. and Goddard, N.J. (1998) Acute percutaneous scaphoid fixation. A pilot study. The Journal of Bone \& Joint Surgery, 80, 95-99. doi:10.1302/0301-620X.80B1.8076

[11] Langhoff, O. and Andersen, J.L. (1988) Consequences of late immobilization of scaphoid fractures. The Journal of Bone \& Joint Surgery, 13, 77-79.

[12] Leslie, I.J. and Dickson, R.A. (1981) The fractured carpal scaphoid. Natural history and factors influencing outcome. The Journal of Bone \& Joint Surgery, 63-B, 225-230.

[13] McQueen, M.M., Gelbke, M.K., et al. (2008) Percutaneous screw fixation versus conservative treatment for fractures of the waist of the scaphoid: A prospective randomised study. The Journal of Bone \& Joint Surgery, 90, 66-71. doi:10.1302/0301-620X.90B1.19767

[14] Geissler, W.B., Adams, J.E., et al. (2012) Scaphoid fractures: What's hot, what's not. The Journal of Bone \& Joint Surgery, 94, 169-181. doi:10.2106/JBJS.942icl 